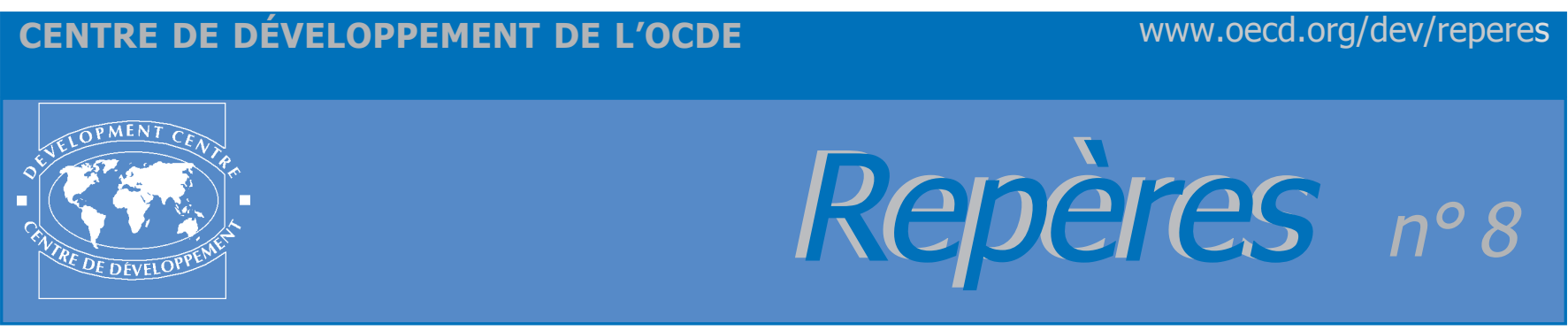

\title{
Énergie et pauvreté en Afrique
}
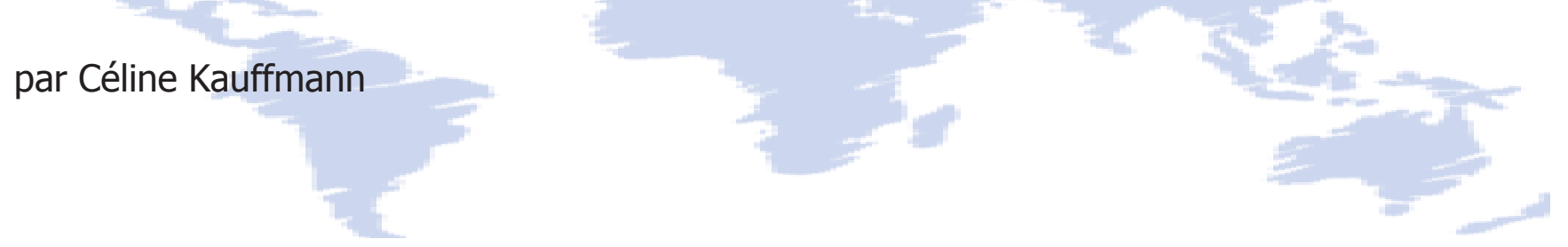

Repères $n^{\circ} 8$ découle des Perspectives économiques en Afrique 2003/2004, une co-édition de la Banque africaine de développement et le Centre de développement de l'OCDE www.oecd.org/dev/perspectivesafricaines

L'accès à l'énergie est une composante essentielle du développement économique, social et politique. Il favorise le développement individuel via l'amélioration des conditions éducatives et sanitaires. Il permet le développement de l'activité économique par la mécanisation et la modernisation des communications. Il participe enfin à l'amélioration de l'environnement économique en permettant une intervention publique plus efficace, un meilleur respect de l'environnement et le renforcement de la démocratie. Cependant, malgré un potentiel énorme en énergies fossiles et renouvelables, I'Afrique présente des déficits énergétiques importants. Les ressources du continent sont sous-exploitées, ou exportées sous forme brute, ou bien encore gaspillées lors de l'extraction ou du transport. En conséquence, l'offre disponible pour les populations est largement insuffisante et la consommation d'énergie s'articule essentiellement autour de la biomasse.

\section{Un vaste potentiel énergétique...} largement inexploité

Les sources d'énergie renouvelable sont nombreuses en Afrique. Les bassins hydrauliques d'Afrique centrale, la faille de la Rift Valley, ainsi que l'ensoleillement dont bénéficie le continent en général sont des sources d'énergies hydraulique, géothermique et solaire peu égalées dans le reste du monde. A l'heure actuelle, néanmoins, seule une infime partie de ce potentiel est exploité : 7 pour cent seulement des capacités hydrauliques, moins de 1 pour cent des capacités géothermiques, et les initiatives photovoltaïques restent encore embryonnaires. Si les énergies fossiles donnent lieu en Afrique à une exploitation plus intensive que les énergies renouvelables, leur consommation domestique reste extrêmement réduite. Ainsi, les trois quarts de la production de pétrole du continent sont destinés à l'exportation. Lorsqu'elles ne sont pas sousexploitées ou exportées, les ressources énergétiques du continent sont gaspillées, ce qui est le cas notamment du gaz, en raison d'infrastructures largement déficientes.

Tous les pays africains ne sont cependant pas logés à la même enseigne. Les ressources énergétiques du continent se répartissent dans des zones distinctes. Le pétrole et le gaz se situent essentiellement en Afrique du Nord et dans les pays riverains du Golfe de Guinée ; I'Afrique australe bénéficie de la quasi-totalité du potentiel de charbon du continent ; les capacités géothermiques se concentrent en Afrique de l'Est et les bassins hydrauliques en Afrique centrale ; le rayonnement solaire est plus fort dans les pays sahéliens... Face à l'hétérogénéité du potentiel, les capacités d'exploitation sont aussi largement inégales. L'Afrique du Nord connaît moins de déperditions lors de I'exploitation du gaz et du pétrole que les pays du Golf de Guinée. Elle présente aussi des capacités de raffinage plus importantes que ses voisins d'Afrique Sub-Saharienne. Ainsi, environ la moitié du pétrole produit localement se retrouvent dans l'offre disponible, contre 11 pour cent au Nigeria, 7.5 pour cent dans les pays d'Afrique Centrale et à peine 5 pour cent en Angola. 


\section{Potentiel et offre d'énergie en Afrique en chiffres}

\section{Énergies renouvelables}

- 21 pays sur 53 seraient en mesure d'exploiter de façon rentable l'énergie hydraulique en Afrique, mais seuls 7 pour cent de ce potentiel est exploité (essentiellement en Egypte, au Mozambique, en Zambie, au Nigéria et au Ghana).

- La Rift Valley pourrait permettre l'accès à un potentiel d'énergie géothermique de l'ordre de 6.5 gigawatts, mais à ce jour, seul le Kenya en fait une exploitation significative (de l'ordre de 121 megawatts).

- Le rayonnement solaire moyen en Afrique s'étale entre 5 et $7 \mathrm{kWh} / \mathrm{m}^{2}$ par jour, à égalité avec la péninsule arabique, le nord de l'Australie et le nord du Chili. Cependant, I'Afrique ne possède que 1.3 pour cent des capacités de production photovoltaïques mondiales.

\section{Biomasse}

- Environ 9 personnes sur 10 en Afrique subsaharienne utilisent la biomasse (bois, résidus...) pour s'éclairer, cuisiner et se chauffer.

- 6 femmes africaines sur 10 vivant en milieu rural sont confrontées à la raréfaction des ressources en bois, contre 8 en Asie et 4 pour cent en Amérique latine.

\section{Pétrole}

- L'Afrique détient 7.3 pour cent des réserves mondiales de pétrole et représente 10.2 pour cent de la production mondiale, mais ne possède que 3.6 pour cent des capacités de raffinage installées dans le monde.

- L'Afrique participe seulement à 3 pour cent de la consommation mondiale de pétrole.

\section{Charbon}

- L'Afrique du Sud possède $9 / 10^{\text {ème }}$ des réserves africaines de charbon, soit 5 pour cent des réserves mondiales.

\section{Électricité}

- Avec 35.5 pour cent de la population reliée à l'électricité en 2002, l'Afrique présente les taux d'électrification les plus faibles du monde en voie de développement (42.8 pour cent en Asie du Sud, 89.2 pour cent en Amérique latine, 88.1 pour cent en Asie de l'Est et 91.8 pour cent au Moyen-Orient).

- Il existe des disparités importantes de taux d'électrification entre I'Afrique du Nord (électrifiée à 93.6 pour cent) et l'Afrique Sub-saharienne (23.6 pour cent).

- L'AIE estime qu'en 2030, les pays en développement devraient être électrifiés à plus de 95 pour cent, en dehors de l'Asie du Sud (66 pour cent) et de l'Afrique Sub-saharienne (51 pour cent).

- En Afrique Sub-saharienne, les populations rurales sont les plus mal loties puisque seuls 8.4 pour cent ont accès à l'électricité.

- 11.3 pour cent de l'électricité offerte en Afrique est gaspillée lors de la production et du transport contre 9.2 pour cent dans le monde. Ces pertes dépassent 20 pour cent au Sénégal, au Kenya et en Tanzanie et 40 pour cent au Nigeria et au Congo.

Source : Banque africaine de développement et le Centre de développement de I'OCDE, Perspectives économiques en Afrique (2003-2004), mise à jour 2005. 
Une consommation très faible, dominée par I'utilisation de la biomasse

En l'absence d'une offre satisfaisante, la consommation énergétique par habitant est très faible en Afrique. Elle est en moyenne de 0.5 tonnes équivalent pétrole par habitant contre 1.2 en moyenne mondiale. Elle se compose essentiellement de l'exploitation de la biomasse, des dérivés pétroliers et de l'électricité, énergie secondaire issue des sources fossiles et des énergies renouvelables. Cependant, les sources d'énergie modernes (produits pétroliers et énergie électrique) sont essentiellement destinées aux usages industriels et au transport. La consommation domestique africaine d'énergie se résume donc en grande partie à l'utilisation de la biomasse.

Le bois et ses dérivés constituent les sources d'énergie les plus utilisées par les ménages africains - surtout ruraux en raison de leur disponibilité et de l'absence de droits de propriété privée sur les ressources forestières, qui en font une ressource quasi gratuite au niveau individuel. Cependant, la disponibilité de ces combustibles diminue largement dans certaines zones en raison de leur surexploitation, obligeant les femmes et les enfants à couvrir des distances de plus en plus longues pour la collecte. De plus, le faible rendement calorifique de la biomasse augmente de beaucoup le coût de son utilisation par calorie consommée. Enfin, les mauvaises conditions de combustion entraînent non seulement un faible rendement mais constituent aussi un danger pour la santé des populations (elles contribuent notamment à la pollution intérieure des maisons, sources de maladies respiratoires graves).

La faiblesse de la consommation d'énergie s'accompagne d'un recours limité à l'électricité. Le continent africain présente le taux d'électrification le plus faible au monde, en dessous des taux de l'Asie du Sud, de l'Amérique latine et du Moyen-Orient. La faible densité de population combinée à une prépondérance de la population rurale sont des contraintes majeures car elles rendent le développement des infrastructures d'électricité très onéreux et limitent les économies d'échelle. De plus, en raison du manque d'entretien, des branchements illégaux et de l'insuffisance des investissements, l'offre est peu fiable, comme en témoignent les fortes pertes lors du transport et de la distribution. L'étroitesse du réseau et son mauvais état général sont des problèmes d'autant plus difficiles à surmonter que les pays africains connaissent de fortes contraintes budgétaires.
Combiner les approches locales, nationales et régionales pour améliorer l'offre d'énergie finale

Face au constat alarmant de l'absence d'une offre d'énergie satisfaisante, les pays africains ont entrepris au cours de la dernière décennie de réformer le mode de propriété, I'organisation et la réglementation du secteur de l'énergie. Plus de 30 pays africains ont notamment amorcé un programme d'ouverture du secteur de l'électricité aux opérateurs privés. Bien que le recul manque pour une évaluation satisfaisante, il semble que ces réformes aient donné des résultats mitigés pour le moment. Dans certains pays, l'existence d'une réglementation fiable a permis d'attirer des investisseurs et d'améliorer la desserte de la population. Dans beaucoup d'autres, un processus incomplet a compromis les chances de réussite, mettant en exergue l'importance cruciale du cadre de régulation pour faciliter le transfert au secteur privé.

Les expériences passées soulignent le rôle essentiel des étapes initiales de la réforme, à savoir la formulation claire d'une politique de l'électricité énonçant les principes directeurs des programmes de réforme et la mise en place d'une instance de contrôle transparente et indépendante pour le marché de l'électricité. L'autorité de régulation vise à faire respecter le cahier des charges afin d'assurer le fonctionnement efficace de l'entreprise en garantissant un élargissement de l'accès à l'électricité. Cela implique une détermination stratégique des prix du service tels qu'ils ne deviennent pas prohibitifs pour les consommateurs, mais qu'ils permettent au repreneur d'effectuer les investissements nécessaires à l'extension du réseau. Seulement dans ce cas, une expansion de la desserte électrique a pu être observée, comme en Côte d'Ivoire.

La réforme nationale doit aussi s'accompagner d'une approche transfrontalière. En effet, la nature des projets d'électricité, notamment leur taille et les possibilités d'économies d'échelle qui leur sont liées, justifie l'extension des réseaux au delà des frontières. Une interconnexion des réseaux est déjà à l'œuvre en Afrique du Nord, en Afrique australe et en voie d'être finalisé en Afrique de I'Ouest. L'Afrique centrale et l'Afrique de I'Est restent cependant largement enclavées. Favoriser les initiatives régionales en faveur d'une amélioration de l'accès à l'électricité constitue à juste titre l'une des propositions fondamentales de l'initiative Énergie du Nepad, qui entend se baser sur le projet INGA pour assurer les interconnections. Là encore, la mise en place d'un cadre régulateur commun adéquat reste une condition cruciale de succès. 


\section{Figure 1. L'accès à l'énergie, clef de voûte du développement économique, social et politique}

L'accès à une offre d'énergie de qualité permet d'améliorer substantiellement les conditions de vie des populations car elle favorise la lutte contre la faim et la malnutrition grâce à la préservation des aliments par la réfrigération, à l'amélioration de la productivité au sein de la chaîne alimentaire et au développement de mode de production agricole modernes. Il constitue un élément essentiel de progrès sanitaire via I'amélioration de l'hygiène alimentaire et le perfectionnement des équipements médicaux.

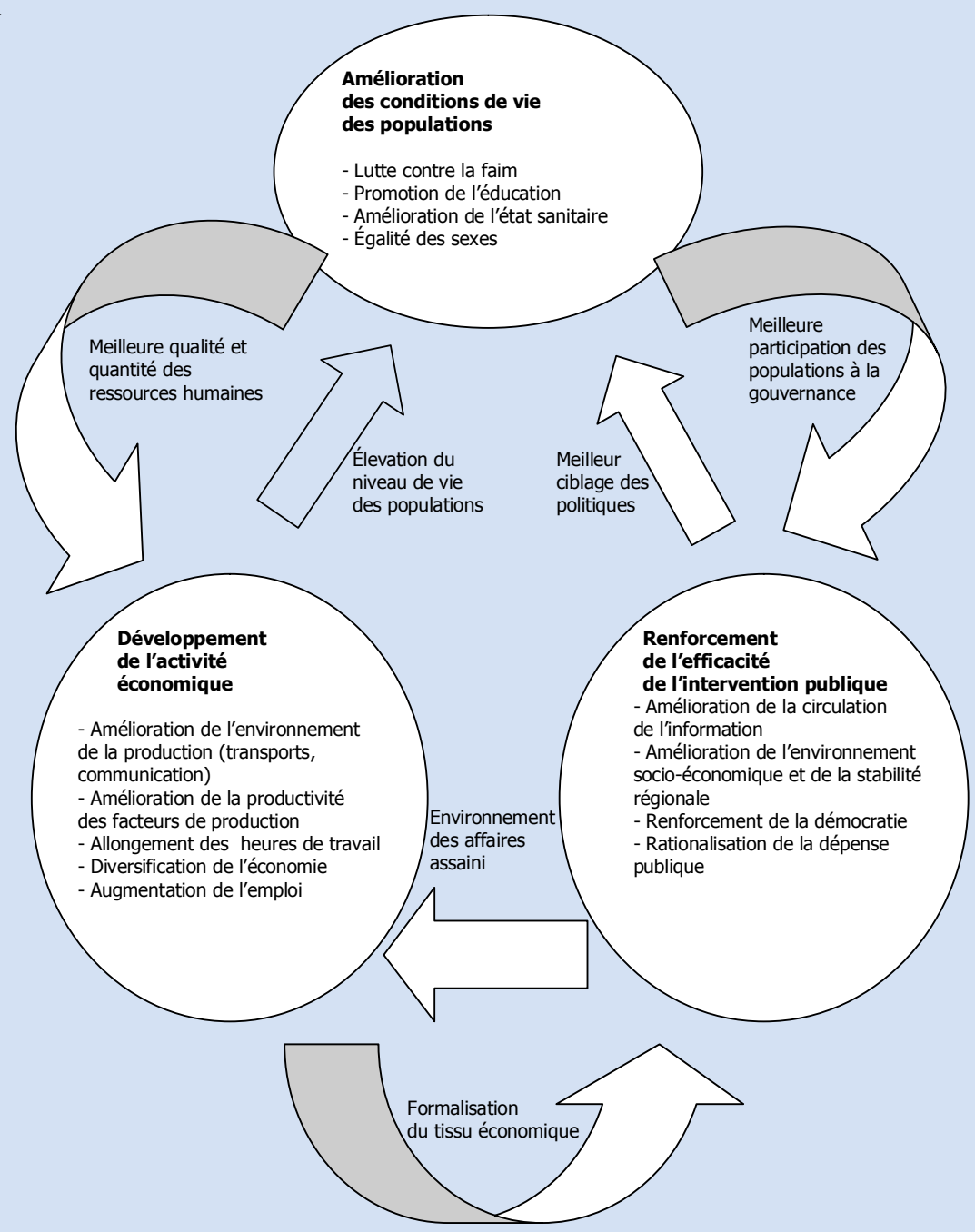

Les énergies modernes sont moins dangereuses et leur utilisation limite la collecte de bois et l'approvisionnement en eau à longue distance souvent physiquement très éprouvants et longs pour les populations. La disponibilité de sources modernes d'énergie permet de généraliser la scolarisation en libérant du temps pour que les enfants suivent l'école et en permettant le développement des cours du soir et du travail tardif à la maison.

A son tour, le progrès des conditions de vie des populations a un impact direct sur le développement de l'activité économique par l'augmentation de l'espérance de vie et l'amélioration de la santé des populations et par une meilleure formation de la main-d'œuvre. Une meilleure offre d'énergie permet aussi l'allongement les heures de travail et la limitation des périodes d'arrêt forcé de l'activité dues aux délestages en électricité. Le recours à des sources d'énergie fiables permet de mieux utiliser les machines et est une condition sine qua non de l'adoption de nouvelles technologies propres à favoriser la diversification des pays. Finalement, l'environnement des affaires bénéficie directement des améliorations enregistrées dans les secteurs clés du transport et de la communication, qui dépendent largement de l'offre d'énergie.

Une meilleure offre d'énergie permet aussi à l'État d'offrir des services d'éducation, de santé et de communication à meilleur coût et en plus grande quantité. Elle favorise la circulation de l'information, élément essentiel de la prise de décision politique. Elle permet de cibler les populations dans le besoin et de faire un choix éclairé des politiques les mieux adaptées au contexte local et national. Réciproquement, la circulation de l'information et l'amélioration des conditions de vie des populations favorisent le développement de la participation de la population aux choix nationaux, pouvant permettre un approfondissement du caractère démocratique des institutions. Les autorités sont ainsi poussées à plus de transparence et de responsabilité dans leurs décisions.

Source : Banque africaine de développement et Centre de développement de I'OCDE, Perspectives économiques en Afrique (2003/2004), figure 12 dans la Vue d'ensemble. 
L'adoption d'une réforme nationale visant à rationaliser l'activité des opérateurs historiques et la mise en place d'une concertation régionale pour le développement des projets à grande échelle sont des conditions nécessaires pour améliorer et étendre les réseaux existants. Cependant, si la plus forte densité des villes laisse à penser qu'elles seront prioritaires pour les futurs programmes d'électrification, près de la moitié de la population africaine devrait continuer à vivre en milieu rural à I'horizon 2025, avec peu ou pas d'accès au réseau national d'électricité. Mettre à disposition de ces populations des modes d'énergie fiables et efficients requiert la recherche de solutions locales flexibles et innovantes. C'est la condition nécessaire d'une amélioration des conditions de vie des populations, mais aussi d'une exploitation soutenable et durable des ressources forestières des pays. Cela passe certainement par une utilisation plus efficace de la biomasse, par le biais notamment de technologies moins polluantes, mais aussi par l'adoption de nouvelles sources d'énergie telles le GPL pour les ménages. Dans les domaines agricoles, des systèmes de production légers, hors réseaux ou dans le cadre de mini réseaux, pourraient être employés tels les micro projets hydrauliques pour l'irrigation, les systèmes de pompage liés à l'énergie solaire ou éolienne et I'utilisation des résidus agricoles pour la (co-)génération d'énergie. Une partie de ces activités pourrait être prise en charge par de petites ou moyennes entreprises, ce qui permettrait de dynamiser l'activité privée locale.

Le succès de ces approches requiert la mise en place par les autorités d'une politique intégrée de l'énergie, favorisant à la fois l'amélioration des structures et infrastructures existantes, la promotion d'initiatives nouvelles en faveur des populations défavorisées et la coopération régionale. Les moyens financiers nécessaires à une amélioration de l'offre d'énergie sont colossaux. Le recours à l'initiative privée là où les bénéfices peuvent être mutuels est certainement une dimension de la solution. Cependant, il ne sera pas suffisant à pallier à la faiblesse des ressources locales, notamment pour le financement des grandes infrastructures et l'extension de l'offre d'énergie en milieu rural. Si la réduction de la pauvreté reste une priorité affichée de la communauté internationale, il est essentiel que l'élargissement de l'offre d'énergie redevienne une priorité des bailleurs de fonds bilatéraux et multilatéraux.

Figure 2. Consommation d'énergie en Afrique et dans le monde en 2002, par source (chiffres Monde)

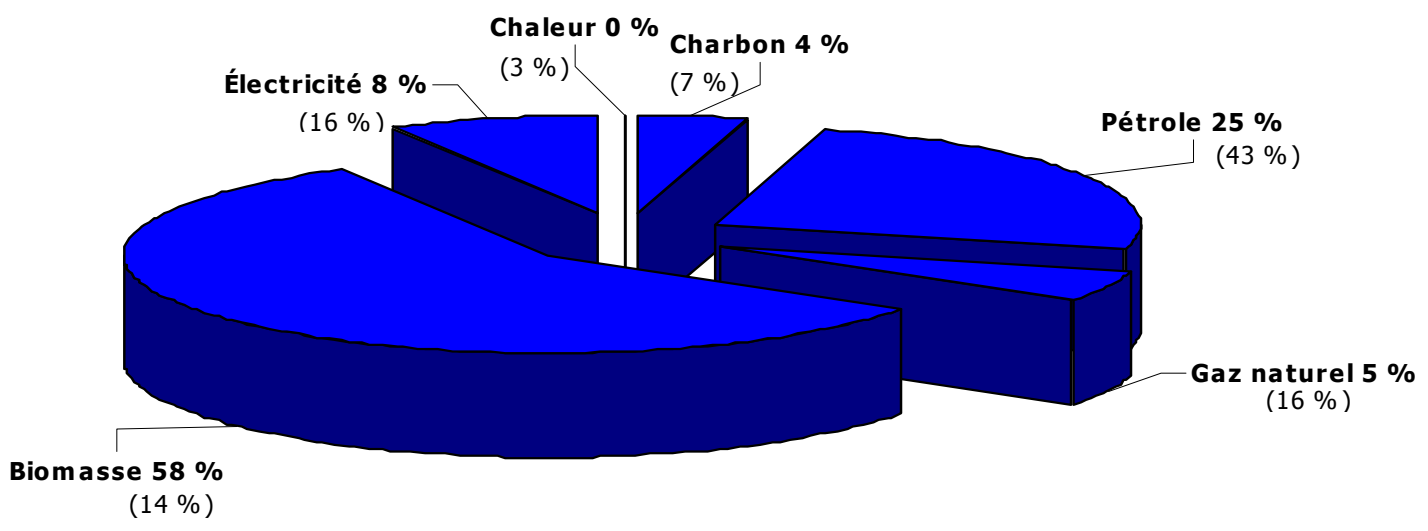

Source : Agence International de l'Énergie. 
Pour consulter les Repères du Centre de développement

www.oecd.org/dev/reperes

les Cahiers de politique économique

www.oecd.org/dev/cahiers

ou les nouveaux Documents de travail

www.oecd.org/dev/dt

\section{OECD $\ll O$}

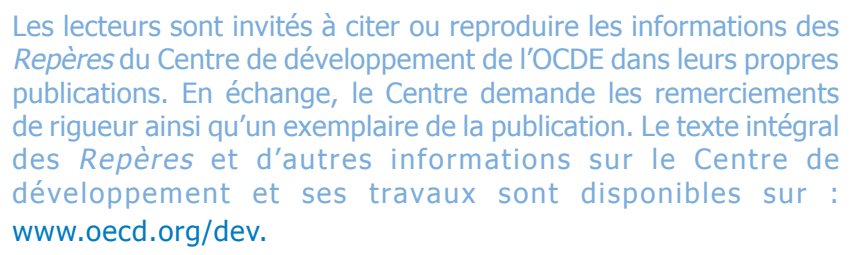

Centre de développement de I'OCDE « Le Seine St Germain »

12 boulevard des Iles

92130 Issy-les-Moulineaux, France

Tél : $33(0) 145.24 .82 .00$

Fax : 33 (0)1 44.30.61.49

mél : cendev.contact@oecd.org 